\title{
The effects of repetitive task training combined with neuromuscular electrical stimulation on extremities for acute cerebral paralysis
}

\author{
Xingnan Liu ${ }^{1,2}$ and Chunli Mei ${ }^{1, a}$ \\ ${ }^{1}$ Department of Nursing, Beihua University, Jilin, 132013, China \\ ${ }^{2}$ The Central Hospital of Jilin City, Jilin, 132013, China
}

\begin{abstract}
In order to study the effect of repetitive task training combined with neuromuscular electrical stimulation on lower limb function for severe paralysis patients with acute cerebral apoplexy. Chose 36 cases of severe paralysis in patients with acute cerebral apoplexy and devided into treatment group and control group. 18 cases in each group, the control group was given routine rehabilitation therapy. The treatment group was given repetitive task training on the basis of control group. Including sit - stand and stand training. Evaluate the lower limb motor function by the FMA, the walking function by FAC and the activities of daily living by FIM respectively before and after 2 months of treatment.The results were showed that the scores of FMA and FIM were significantly better than before treatment after 2 months $(\mathrm{P}<$ $0.05)$, and the the scores of FMA and FIM in treatment group were better than control group (P $<0.01$ ). The scores changed of FMA and FIM in treatment group ( $\triangle$ FMA and $\triangle$ FIM scores) were better than control group $(\mathrm{P}<0.01)$.Walking function was better in treatment group than control group as the grade of FAC in treatment group was more higher. So repetitive task training combined neuromuscular electrical stimulation can more effectively improve the lower limb exercise function, walking function and daily life activity for severe paralysis patients with acute cerebral apoplexy.
\end{abstract}

\section{Introduction}

At present, there are few studies on the early rehabilitation therapy for severe paralysis in patients with cerebral apoplexy. In recent years, the research showed that repetitive task training can improve the walking ability of patients with cerebral apoplexy[1]. Neuromuscular electrical stimulation also could promote the recovery of motor function of paralyzed limbs[2].

The purpose of this study is to investigate the effect of repetitive task training combined with neuromuscular electrical stimulation on lower limb function for severe paralysis in patients with acute cerebral apoplexy.

\footnotetext{
${ }^{\mathrm{a}}$ Corresponding author: gaoda2002@126.com

The project supported by educational department and technology department of Jilin province in China. (No. 2015153, 2013ZC029, 201339033).
} 


\section{Materials and Methods}

\subsection{Object of study}

36 patients were selected with acute large area cerebral infarction in neurology department and stroke unit from affiliated hospital of Beihua university from June 6, 2014 to August 2015. Divided randomly into group of training task combined with electrical stimulation therapy(treatment group) and group of electrical stimulation treatment (control group) with 18 cases in each group.

Inclusion criteria: acute patients with large area cerebral infarction in middle arterial blood supply area (atherosclerotic thrombotic cerebral infarction) and there were clearly responsibility of infarction lesions on head CT or MRI. With first time occurrence and patients were under acute phase(course of disease $<7 d$ ). Vital signs of patients were relatively stable, and patients were willing to cooperate with treatment as the disease condition was not getting worse. In the presence of severe limb paralysis and lower extremity muscle strength level was from 0 - 1. Patients were willing to sign informed consent.

Exclusion criteria: High mortality of patients with MCA, including the range of infarction was more than that of middle cerebral artery and the patients were under coma state. Or the patients had serious heart, lung, kidney disease who were not suitable for early rehabilitation therapy; Patients with severe pain of lower limb who could not complete treatment. Patients had cognitive or mental disorders who can not cooperate with treatment. The treatment efficacy can not be judged as patients who had poor compliance and did not comply with regulation treatment[3].

\subsection{Therapeutic methods}

Control group: based on conventional rehabilitation therapy including neural facilitation techniques, electroacupuncture, electric standing bed, etc. Enhanced neuromuscular electrical stimulation therapy was given to paralysis limb extensor (femoral quadriceps and tibialis anterior muscle group). The first week of treatment: 20 minutes per time, 1 time per day. The second week of treatment: 40 minutes per time, 1 time per day. The third week of treatment: 40 minutes per time, 2 times per day. Task training combined with electrical stimulation treatment group (treatment group): on the basis of the control group treatment, increase repetitive task training. First week of treatment: 10 minutes per day, 1 time per day. At the second week: 20 minutes per time, 1 time a day; At the third weeks: 20 minutes per time, 2 times a day. Given repetitive task training for relatively stable conditioned patients. We found that brain edema was reducing, consciousness getting better, blood pressure, breathing and heart rate were relatively stable. Given seat training in first week, lifting bed seat training, bed edge seat training and wheelchair seat training, repeat 3 times a day. The time were 2 weeks.sit-stand training: to begin with, the seat should be high, patient easy to stand up, which can be in a wheelchair, stool or a treatment bed, while standing, the patients' arms should place in the bed bar or treating bar. Treatment staff or family members helped patients stand up or keep standing. Place of treatment can be in the ward or treatment rooms, the treatment needed family members in, repeats 15 times a day. Observed the patient's condition, the patients tolerance range and the procedure shall be slowly. All patients selected should be treated with conventional nerve nutrition according to the condition and improve therapy of circulation, dehydration, anti-platelet aggregation[4].

\subsection{Clinical evaluation}

Records of general situation, including the systemic complications of patients, etc. Treatment records: the specific treatment time, completion of the situation, etc. Records of adverse effects or adverse events: including whether the patient's condition was getting worse or not, blood pressure, heart rate changing, muscle fatigue, falling down, etc. Evaluate the function of exercise by the FMA, divided 
into 17 items, a total of 34 points, the recovery of motor function of lower limb was getting better as the score was getting higher[5]. Evaluate the walking function by FAC, it was consisted of six level which from 0 to 5, the ability of walking was getting better as the level was getting higher. Evaluated the activities of daily living by FIM, using seven point scale, the independence was getting better and the dependence was getting smaller as the score was getting higher. Evaluate clinic and recovery of patients before treatment and during two months of treatment. All evaluation items shall be carried out by the trained personnel.

\subsection{Statistical analysis}

SPSS software was used for statistical analyses and the values are presented as the means +/- SD. ANOVA was used to compare the mean values. $\mathrm{P}$ values of less than 0.05 were taken to indicate statistically significant differences.

\section{Results}

The two groups of patients had not appear disease aggravation, recurrent stroke, cardiovascular accident, falling down and other adverse events during therapeutic process, 2 cases in the treatment group and 3 cases in the control group appeared mild dizziness and headache during the treatment, both treatment group and control group had 2 cases who appeared limb pain and other symptoms of muscle fatigue, the symptoms were alleviated after symptomatic treatment. FMA and FIM scores of these two groups had no significant difference before treatment $(\mathrm{P}>0.05)$. After 2 months treatment, the scores were significantly better than before $(\mathrm{P}<0.05)$, FMA and FIM scores of treatment group were significantly better than control group $(\mathrm{P}<0.01)$; FMA and FIM scores changed quantity of the treatment group $(\triangle \mathrm{FMA}$ and $\triangle \mathrm{FIM}$ scores) were significantly better than control group $(\mathrm{P}<0.01)$ after further treatment. FAC grade of treatment group was higher, FAC grading differences between these two groups had significant meaning $(\mathrm{P}<0.05)$ after 2 months treatment, See Table 1.

Table 1. Two groups of patients before and after treatment of FMA, FIM score

\begin{tabular}{|c|c|c|c|c|c|c|}
\hline \multirow{2}{*}{ Score } & \multicolumn{3}{|c|}{ FMA } & \multicolumn{3}{c|}{ FIM } \\
\cline { 2 - 7 } & $\begin{array}{c}\text { Control } \\
\text { Group }\end{array}$ & $\begin{array}{c}\text { Treatment } \\
\text { Group }\end{array}$ & P Value & $\begin{array}{c}\text { Control } \\
\text { Group }\end{array}$ & $\begin{array}{c}\text { Treatment } \\
\text { Group }\end{array}$ & P Value \\
\hline 0 month & $3.78 \pm 1.48$ & $3.83 \pm 1.38$ & 0.06 & $27.30 \pm 6.30$ & $28.60 \pm 6.30$ & 0.07 \\
\hline 1 month & $18.11 \pm 3.66$ & $24.06 \pm 3.90$ & 0.04 & $18.11 \pm 3.66$ & $68.67 \pm 13.09$ & 0.03 \\
\hline 2 months & $14.33 \pm 3.40$ & $20.22 \pm 4.35$ & 0.01 & $23.67 \pm 13.5$ & $40.11 \pm 12.22$ & 0.01 \\
\hline
\end{tabular}

\section{Discussion}

Many foreign studies had confirmed that the treatment effect was closely related to the treatment time.The quantity of patients with cerebral apoplexy, the treatment and effect of acute and subacute phase had independent dose dependent relationship, high intensity and repetitive rehabilitation could improve the motor function and the reorganization of the cerebral cortex of the patients[6].At present, many scholars at home and abroad think that the key factor for the effective rehabilitation therapy after cerebral apoplexy is to carry on the repetition, the task oriented training, and the training will be high strength and long time.

In recent years, some high quality meta analysis in foreign countries showed: within 6 months of the onset of the disease, repetitive task oriented training can improve the walking ability of cerebral apoplexy patients (including walking distance and walking speed) and ADL [7]. Foreign experts had recommend taking repetitive task oriented training, evidence based on medical class A and B. Study had proved that it was similar that neuromuscular electrical stimulation therapy which had been used wildly during the treatment of cerebral apoplexy rehabilitation can promote the recovery of motor and 
sensory function, and the dose of electrical stimulation was an important factor for the recovery of limb function, benefit of motor function would be greater as the duration time of the stimulus was getting longer for patients with severe paralysis[8].

In this study, control group used the treatment plan of strengthening electrical stimulation of nerve muscle for severe paralysis patients with acute cerebral apoplexy which based on clinical and research results and scientific theory in domestic and overseas. The stimulation time was $20 \mathrm{~min} / \mathrm{d}$, and then increase 40 to $80 \mathrm{~min} / \mathrm{d}$. Treatment group adopt treatment methods of repetitive task orientation training combined with intense neuromuscular electrical stimulation. The results showed that the two groups of patients did not appear the stroke recurrence, disease progression and cardiovascular accidents, fell down, adverse events in the process of treatment, these situation can prove that early treatment was safe and patients can tolerate. Walking function and activities of daily living (ADL) were significantly improved of two groups of patients with lower extremity motor function after 2 months of treatment, but the benefit of the treatment group was greater than that of the control group, lower limb motor function was better, walking function classification was higher, more proportion of body achieved the walking function and the ability of daily living. The biggest task was to achieve the goal of walking function as far as possible for severely paralyzed patients with acute cerebral apoplexy, patients can not be directly carried out walking training for poor muscle strength. While sitting, standing ability and sit-stand transfer ability is the foundation to realize the walking function of patients in the future[9-10]. Treatment group used repeat seat training, sit-stand training, standing level of training and oriented task training step by step and combined with stimulation treatment of increased neuromuscular electrical stimulation to effectively improved lower limb function in advance and created a key precondition for realizing further walking function. The most important was that patients and their families who accepted the treatment learned method of self training and they was encouraged by benefit from initial treatment. Patients can keep family training with the high positivity even leaving hospital, therefore, long-term benefits can be obtained as treatment can be extended.

Combined drug therapy and early rehabilitation therapy, The state of illness will be severe for the acute cerebral apoplexy patients with large area infarction, the dehydration, nutrition, improvement of circulation, anti platelet aggregation drugs and some other drugs shall be needed in the early stage. At the same time, blood pressure, blood sugar and heart and lung function of the patient may not be stable, therefore bedside rehabilitation therapy shall be the main treatment within 1week and treatment volume shall not be large because some patients appeared small amount of hemorrhage after secondary infarction. Take active rehabilitation therapy gradually after that the vital signs of patient is stable, carry out Staged task oriented training, and strengthen the treatment of nerve muscle electrical stimulation, but the treatment plan still need to be adjusted according to the patient's condition during treatment process.

In conclusion, the preliminary clinical study confirmed that limb motor function, walking function can be improved by adopting the therapeutic schedule of repeated task oriented training combined with neuromuscular electrical stimulation. So the ability of daily life will be improved. There are still many deficiencies in this study. For example: selected samples are less, the follow-up time is shorter. Failure to comply with the requirements of ICF to conduct a comprehensive evaluation of efficacy from three levels etc. These problems will be improved in the future research.

\section{References}

1. B.French, L.Thomas, M. Leathley, et al. Does repetitive task training improve functional activity after stroke? A Cochrane systematic review and meta-analysis[J], J Rehabil Med.42(1):914.(2010).

2. JM.Veerbeek, M.Koolstra ,JC.Ket, et al. Effects of augmented exercise therapy on outcome of gait and gait-related activities in the first 6 months after stroke: a meta-analysis[J], Stroke.42(11):3311-3315.(2011).

3. L.Peter, B.Julie,K.Gert. Stroke rehabilitation[J], Lancet.377: 1693-1702.(2011). 
4. SS.Hsu, MH.Hu, YH.Wang, et al. Dose-response relation between neuromuscular electrical stimulation and upper-extremity function in patients with stroke[J], Stroke.41(4):821-824.(2011).

5. SS.Hsu, MH.Hu, JJ.Luh, et al. Dosage of neuromuscular electrical stimulation: is it a determinant of upper limb functional improvement in stroke patients[J],J Rehabil Med.44(2):125-130.(2012).

6. AR.Fugl-Meyer, L.Jaasko, I.Leyman, et al. The poststroke hemiplegic patient. a method for evaluation of physical performance[J],Scand J Rehabil Med.7:13-31.(1975).

7. MK.Holden, KM.Gill, MR.Magliozzi , et al. Clinical gait assessment in the neurologically impaired. Reliability and meaningfulness[J],Phys Ther.64(1):35-40.(1984).

8. BE.Bates, MG.Stineman . Outcome indicators for stroke: application of an algorithm treatment across the continuum of postacute rehabilitation services[J],Arch Phys Med Rehabil.11:14681478.(2000).

9. JM.Veerbeek, G.Kwakkel, EE.van Wegen, et al. Early prediction of outcome of activities of daily living after stroke: a systematic review[J],Stroke.42(5):1482-1488.(2011).

10. B.French, LH.Thomas, MJ.Leathley, et al. Repetitive task training for improving functional ability after stroke[J], Cochrane Database Syst Rev.10(4).(2007). 